\title{
REGULARITY OF SOLUTIONS TO THE NAVIER-STOKES EQUATIONS IN $\dot{B}_{\infty, \infty}^{-1}$
}

\author{
GREGORY SEREGIN AND DAOGUO ZHOU
}

\begin{abstract}
We prove that if $u$ is a suitable weak solution to the three dimensional Navier-Stokes equations from the space $L_{\infty}\left(0, T ; \dot{B}_{\infty, \infty}^{-1}\right)$, then all scaled energy quantities of $u$ are bounded. As a consequence, it is shown that any axially symmetric suitable weak solution $u$, belonging to $L_{\infty}\left(0, T ; \dot{B}_{\infty, \infty}^{-1}\right)$, is smooth.
\end{abstract}

\section{INTRODUCTION}

The main aim of this paper is to show that suitable weak solutions to the NavierStokes equations, whose $\dot{B}_{\infty, \infty}^{-1}$-norm is bounded, have the Type I singularities (or Type I blowups) only. To be more precise in the statement of our results, we need to define certain notions.

Definition 1.1. Let $\Omega$ be a domain in $\mathbb{R}^{3}$ and let $\left.Q_{T}:=\Omega \times\right] 0, T$. It is said that a pair of functions $v$ and $q$ is a suitable weak solution to the Navier-Stokes equations in $Q_{T}$ if the following conditions are fulfilled:

(i) $v \in L_{\infty}\left(\delta, T ; L_{2, l o c}(\Omega)\right) \cap L_{2}\left(\delta, T ; W_{2, l o c}^{1}(\Omega)\right), \quad q \in L_{\frac{3}{2}}\left(\delta, T ; L_{\frac{3}{2}, l o c}(\Omega)\right)$ for any $\delta \in] 0, T]$

(ii) $v$ and $q$ satisfy the Navier-Stokes equations

$$
\partial_{t} v+v \cdot \nabla v-\Delta v=-\nabla q, \quad \operatorname{div} v=0
$$

in $Q_{T}$ in the sense of distributions;

(iii) for $\left.Q\left(z_{0}, R\right) \subset \Omega \times\right] 0, T[$, the local energy inequality

$$
\begin{aligned}
& \int_{B\left(x_{0}, R\right)} \varphi|v(x, t)|^{2} d x+2 \int_{t_{0}-R^{2}}^{t} \int_{B\left(x_{0}, R\right)} \varphi|\nabla v|^{2} d x d \tau \leq \\
\leq & \int_{t_{0}-R^{2}}^{t} \int_{B\left(x_{0}, R\right)}\left(|v|^{2}\left(\partial_{t} \varphi+\Delta \varphi\right)+v \cdot \nabla \varphi\left(|v|^{2}+2 q\right)\right) d x d \tau
\end{aligned}
$$

holds for a.a. $t \in] t_{0}-R^{2}, t_{0}\left[\right.$ and for all non-negative test functions $\varphi \in C_{0}^{\infty}\left(B\left(x_{0}, R\right)\right.$ $\times] t_{0}-R^{2}, t_{0}+R^{2}[$ ).

Let us introduce the following scaled energy quantities:

$$
\begin{gathered}
A\left(z_{0}, r\right):=\sup _{t_{0}-r^{2}<t<t_{0}} \frac{1}{r} \int_{B\left(x_{0}, r\right)}|v(x, t)|^{2} d x, \quad E\left(z_{0}, r\right):=\frac{1}{r} \int_{Q\left(z_{0}, r\right)}|\nabla v|^{2} d x d t, \\
C\left(z_{0}, r\right):=\frac{1}{r^{2}} \int_{Q\left(z_{0}, r\right)}|v|^{3} d x d t, \quad D\left(z_{0}, r\right):=\frac{1}{r^{2}} \int_{Q\left(z_{0}, r\right)}|q|^{\frac{3}{2}} d x d t,
\end{gathered}
$$




$$
\begin{aligned}
G\left(z_{0}, r\right) & :=\max \left\{A\left(z_{0}, r\right), E\left(z_{0}, r\right), C\left(z_{0}, r\right)\right\}, \\
g\left(z_{0}, r\right) & :=\min \left\{A\left(z_{0}, r\right), E\left(z_{0}, r\right), C\left(z_{0}, r\right)\right\} .
\end{aligned}
$$

Here, $\left.Q\left(z_{0}, r\right):=B\left(x_{0}, r\right) \times\right] t_{0}-r^{2}, t_{0}\left[\right.$ and $B\left(x_{0}, r\right)$ is the ball of radius $r$ centred at a point $x_{0} \in \mathbb{R}^{3}$.

The important feature of the above quantities is that all of them are invariant with respect to the Navier-Stokes scaling.

Our main result is as follows.

Theorem 1.2. Let $\Omega=\mathbb{R}^{3}$. Assume that a pair $v$ and $q$ is a suitable weak solution to the Navier-Stokes equations in $Q_{T}$. Moreover, it is supposed that

$$
v \in L_{\infty}\left(0, T ; \dot{B}_{\infty, \infty}^{-1}\left(\mathbb{R}^{3}\right)\right) .
$$

Then, for any $\left.\left.z_{0} \in \mathbb{R}^{3} \times\right] 0, T\right]$, we have the estimate

$\sup _{0<r<r_{0}} G\left(z_{0}, r\right) \leq c\left\{\left[C\left(z_{0}, 1\right)+D\left(z_{0}, 1\right)\right] r_{0}^{\frac{1}{2}}+\|v\|_{L_{\infty}\left(0, T ; \dot{B}_{\infty, \infty}^{-1}\left(\mathbb{R}^{3}\right)\right)}^{2}+\|v\|_{L_{\infty}\left(0, T ; \dot{B}_{\infty}^{-1}, \infty\left(\mathbb{R}^{3}\right)\right)}^{6}\right\}$,

where $r_{0} \leq \frac{1}{2} \min \left\{1, t_{0}\right\}$ and $c$ is an absolute positive constant.

Let us recall one of definitions of the norm in the space $\dot{B}_{\infty, \infty}^{-1}\left(\mathbb{R}^{3}\right)=\left\{f \in S^{\prime}\right.$ : $\left.\|f\|_{\dot{B}_{\infty, \infty}^{-1}\left(\mathbb{R}^{3}\right)}<\infty\right\}$, which is the following:

$$
\|f\|_{\dot{B}_{\infty, \infty}^{-1}\left(\mathbb{R}^{3}\right)}:=\sup _{t>0} t^{\frac{1}{2}}\|w\|_{L_{\infty}\left(\mathbb{R}^{3}\right)}
$$

where $S^{\prime}$ is the space of tempered distributions, $w$ is the solution to the Cauchy problem for the heat equation with initial datum $f$.

Definition 1.3. Assume that $z_{0}=\left(x_{0}, t_{0}\right)$ is a singular point of $v$, i.e., there is no parabolic vicinity of $z_{0}$ where $v$ is bounded. We call $z_{0}$ Type I singularity (or Type I blowup) if there exists a positive number $r_{1}$ such that

$$
\sup _{0<r<r_{1}} g\left(z_{0}, r\right)<\infty .
$$

According to Definition 1.3. any suitable weak solution, satisfying assumption (1.1), has Type I singularities only. In particular, arguments, used in paper [21, show that axially symmetric suitable weak solutions to the Navier-Stokes equations have no Type I blowups. This is an improvement of what has been known so far, see papers [14] and 21], where condition (1.1) is replaced by stronger one

$$
v \in L_{\infty}\left(0, T ; B M O^{-1}\left(\mathbb{R}^{3}\right)\right) .
$$

Regarding other regularity results on axially symmetric solutions to the NavierStokes equations, we refer to papers 2 , 13, 4, 5, 9, 10, 11, 12, 15, 16, 17, 18, 19, 20, $22,23,24$.

Another important consequence is that the smallness of $\|v\|_{L \infty\left(0, T ; \dot{B}_{\infty, \infty}^{-1}\left(\mathbb{R}^{3}\right)\right)}$ implies regularity, see also [1, 7.

\section{Proof of the Main Result}

In this section, Theorem 1.2 is proved. First, we recall the known multiplicative inequality, see [6]. 
Lemma 2.1. For any $u \in \dot{B}_{\infty, \infty}^{-1}\left(\mathbb{R}^{3}\right) \cap \dot{H}^{1}\left(\mathbb{R}^{3}\right)$, the following is valid:

$$
\|u\|_{L_{4}\left(\mathbb{R}^{3}\right)} \leq c\|u\|_{\dot{B}_{\infty, \infty}^{-1}\left(\mathbb{R}^{3}\right)}^{\frac{1}{2}}\|\nabla u\|_{L_{2}\left(\mathbb{R}^{3}\right)}^{\frac{1}{2}},
$$

where $\dot{H}^{1}\left(\mathbb{R}^{3}\right)$ is a homogeneous Sobolev space.

In fact, a weaker version of (2.1) with $\|u\|_{L^{4, \infty}}$ instead of $\|u\|_{L_{4}\left(\mathbb{R}^{3}\right)}$ is needed. Here, $L^{4, \infty}\left(\mathbb{R}^{3}\right)$ is a weak Lebesgue space. An elementary proof of a weaker inequality is given in 13 .

The second auxiliary statement is about cutting-off in the space $\dot{B}_{\infty, \infty}^{-1}\left(\mathbb{R}^{3}\right)$.

Lemma 2.2. Let $u \in \dot{B}_{\infty, \infty}^{-1}\left(\mathbb{R}^{3}\right)$ and $\phi \in C_{0}^{\infty}\left(\mathbb{R}^{3}\right)$. Then

$$
\|u \phi\|_{\dot{B}_{\infty, \infty}^{-1}\left(\mathbb{R}^{3}\right)} \leq c(|\operatorname{spt} \phi|)\|u\|_{\dot{B}_{\infty, \infty}^{-1}\left(\mathbb{R}^{3}\right)} .
$$

We have not found out a proof of Lemma 2.2 in the literature and presented it in Appendix. Our proof is elementary and based on typical PDE's arguments. A scaled version of the previous lemma is as follows.

Lemma 2.3. For any $u \in \dot{B}_{\infty, \infty}^{-1}\left(\mathbb{R}^{3}\right) \cap H^{1}(B(2))$, the estimate

$$
\|u\|_{L^{4, \infty}(B)} \leq c\|u\|_{\dot{B}_{\infty, \infty}^{-1}\left(\mathbb{R}^{3}\right)}^{\frac{1}{2}}\|u\|_{H^{1}(B(2))}^{\frac{1}{2}} .
$$

is valid for a universal constant c. Moreover, if $u \in \dot{B}_{\infty, \infty}^{-1}\left(\mathbb{R}^{3}\right) \cap H^{1}\left(B\left(x_{0}, 2 R\right)\right)$, then

$$
\|u\|_{L^{4, \infty}\left(B_{R}\left(x_{0}\right)\right)} \leq c\|u\|_{\dot{B}_{\infty, \infty}^{-1}\left(\mathbb{R}^{3}\right)}^{\frac{1}{2}}\left(\|\nabla u\|_{L_{2}\left(B_{2 R}\left(x_{0}\right)\right)}+\frac{1}{R}\|u\|_{L_{2}\left(B_{2 R}\left(x_{0}\right)\right)}\right)^{\frac{1}{2}}
$$

with a universal constant $c$.

Here, we use notation for the ball centred at the origin $B(R)=B(0, R)$ and $B=B(1)$.

Proof. It follows from Lemma 2.1 that for all $\phi \in C_{0}^{\infty}\left(\mathbb{R}^{3}\right)$,

$$
\|u \phi\|_{L^{4, \infty}\left(\mathbb{R}^{3}\right)} \leq c\|u \phi\|_{\dot{B}_{\infty, \infty}^{-1}\left(\mathbb{R}^{3}\right)}^{\frac{1}{2}}\|u \phi\|_{\dot{H}^{1}\left(\mathbb{R}^{3}\right)}^{\frac{1}{2}} .
$$

Taking a cut-off function $\phi$ such that $\phi=1$ in $B, \phi=0$ out of $B(2)$, and $0 \leq \phi \leq 1$ for $1 \leq|x| \leq 2$, we get inequality (2.2) from Lemma 2.2.

To prove inequality (2.3), one can use scaling and shift $x=x_{0}+R y, x \in$ $B\left(x_{0}, 2 R\right), y \in B(2)$ in (2.2).

In order to prove the main result, we need the following auxiliary inequalities for $C\left(z_{0}, r\right)$.

Lemma 2.4. For any $0<r \leq R<\infty$, we have

$$
C\left(z_{0}, r\right) \leq c\|u\|_{L_{\infty}\left(0, T ; \dot{B}_{\infty, \infty}^{-1}\left(\mathbb{R}^{3}\right)\right)}^{\frac{3}{2}}\left(A^{\frac{3}{4}}\left(z_{0}, 2 r\right)+E^{\frac{3}{4}}\left(z_{0}, 2 r\right)\right),
$$

and

$$
C\left(z_{0}, r\right) \leq c\|u\|_{L_{\infty}\left(0, T ; \dot{B}_{\infty, \infty}^{-1}\left(\mathbb{R}^{3}\right)\right)}^{\frac{3}{2}}\left(\frac{R}{r}\right)^{\frac{3}{4}}\left(A^{\frac{3}{4}}\left(z_{0}, R\right)+E^{\frac{3}{4}}\left(z_{0}, R\right)\right)
$$


Proof. Obviously, (2.5) easily follows from (2.4). So, we need to prove the first inequality only. By the Hölder inequality, we have

$$
\|u(\cdot, t)\|_{L_{3}\left(B\left(x_{0}, r\right)\right.} \leq \operatorname{cr}^{\frac{1}{4}}\|u(\cdot, t)\|_{L^{4, \infty}\left(B\left(x_{0}, r\right)\right.}
$$

and thus, by (2.3),

$$
\begin{gathered}
C\left(z_{0}, r\right)=\frac{1}{r^{2}} \int_{t_{0}-r^{2}}^{t_{0}}\|u(\cdot, t)\|_{L_{3}\left(B\left(x_{0}, r\right)\right)}^{3} d t \\
\leq c \frac{1}{r^{\frac{3}{4}}}\|u\|_{L_{\infty}\left(0, T ; \dot{B}_{\infty, \infty}^{-1}\left(\mathbb{R}^{3}\right)\right)}^{\frac{3}{2}}\left(\int_{t_{0}-(2 r)^{2}}^{t_{0}}\|\nabla u(\cdot, t)\|_{L_{2}\left(B\left(x_{0}, 2 r\right)\right)}^{2}+\right. \\
\left.+\frac{1}{r^{2}}\|u(\cdot, t)\|_{L_{2}\left(B\left(x_{0}, 2 r\right)\right)}^{2} d t\right)^{\frac{3}{4}} \\
\leq c \frac{1}{r^{\frac{3}{4}}}\|u\|_{L_{\infty}\left(0, T ; \dot{B}_{\infty, \infty}^{-1}\left(\mathbb{R}^{3}\right)\right)}^{\frac{3}{2}}\left(\int_{t_{0}-(2 r)^{2}}^{t_{0}}\|\nabla u(\cdot, t)\|_{L_{2}\left(B\left(x_{0}, 2 r\right)\right)}^{2} d t+\right. \\
\left.+\sup _{-(2 r)^{2}+t_{0} \leq t<t_{0}}\|u(\cdot, t)\|_{L_{2}\left(B\left(x_{0}, 2 r\right)\right)}^{2}\right)^{\frac{3}{4}} .
\end{gathered}
$$

This completes the proof of inequality (2.4).

Now we are going to jusify our main result.

Proof of Theorem 1.2. From the local energy inequality, it follows that, for any $0<r<\infty$,

$$
A\left(z_{0}, r\right)+E\left(z_{0}, r\right) \leq c\left(C^{\frac{2}{3}}\left(z_{0}, 2 r\right)+C\left(z_{0}, 2 r\right)+D\left(z_{0}, 2 r\right)\right) .
$$

For the pressure $q$, we have the decay estimate

$$
D\left(z_{0}, r\right) \leq c\left(\frac{r}{R} D\left(z_{0}, R\right)+\left(\frac{R}{r}\right)^{2} C\left(z_{0}, R\right)\right) .
$$

which is valid for any $0<r<R<\infty$.

Assume that $0<r \leq \frac{\rho}{4}<\rho \leq 1$. Combining (2.7) and (2.6), we find

$$
\begin{gathered}
A\left(z_{0}, r\right)+E\left(z_{0}, r\right)+D\left(z_{0}, r\right) \leq \\
\leq c\left(C^{\frac{2}{3}}\left(z_{0}, 2 r\right)+C\left(z_{0}, 2 r\right)+\left(\frac{\rho}{r}\right)^{2} C\left(z_{0}, \frac{\rho}{2}\right)+\frac{r}{\rho} D\left(z_{0}, \frac{\rho}{2}\right)\right) .
\end{gathered}
$$

Now, let us estimate each term on the right hand side of the last inequality. From (2.4), (2.5), and Young's inequality with an arbitrary positive constant $\delta$, we can derive

$$
C\left(z_{0}, 2 r\right) \leq c \delta\left(A\left(z_{0}, \rho\right)+E\left(z_{0}, \rho\right)\right)+c \delta^{-3}\|u\|_{L^{\infty}\left(0, T ; \dot{B}_{\infty, \infty}^{-1}\left(\mathbb{R}^{3}\right)\right)}^{6}\left(\frac{\rho}{r}\right)^{3} .
$$

Similarly,

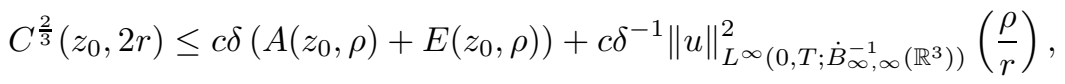

and

$$
\left(\frac{\rho}{r}\right)^{2} C\left(z_{0}, \frac{\rho}{2}\right) \leq c \delta\left(A\left(z_{0}, \rho\right)+E\left(z_{0}, \rho\right)\right)+c \delta^{-3}\|u\|_{L^{\infty}\left(0, T ; \dot{B}_{\infty, \infty}^{-1}\left(\mathbb{R}^{3}\right)\right)}^{6}\left(\frac{\rho}{r}\right)^{8} .
$$


REGULARITY OF SOLUTIONS TO THE NAVIER-STOKES EQUATIONS IN $\dot{B}_{\infty, \infty}^{-1} \quad 5$

Denote $\mathcal{E}(r)=A\left(z_{0}, r\right)+E\left(z_{0}, r\right)+D\left(z_{0}, r\right)$. By a simple inequality $D\left(z_{0}, \rho / 2\right) \leq$ $c D\left(z_{0}, \rho\right)$,

$$
\begin{aligned}
\mathcal{E}(r) \leq & c\left(\delta+\frac{r}{\rho}\right) \mathcal{E}(\rho)+c\left\{\|u\|_{L_{\infty}\left(0, T ; \dot{B}_{\infty, \infty}^{-1}\left(\mathbb{R}^{3}\right)\right)}^{2}\left(\frac{\rho}{r}\right) \delta^{-1}+\right. \\
& \left.+\|u\|_{L_{\infty}\left(0, T ; \dot{B}_{\infty, \infty}^{-1}\left(\mathbb{R}^{3}\right)\right)}^{6}\left[\left(\frac{\rho}{r}\right)^{3}+\left(\frac{\rho}{r}\right)^{8}\right] \delta^{-3}\right\} .
\end{aligned}
$$

Letting $r=\theta \rho$ and $\delta=\theta$ and picking up $\theta$ such that $2 c \theta^{1 / 2} \leq 1$, we find

$$
\mathcal{E}(\theta \rho) \leq \theta^{1 / 2} \mathcal{E}(\rho)+c\left\{\|u\|_{L_{\infty}\left(0, T ; \dot{B}_{\infty, \infty}^{-1}\left(\mathbb{R}^{3}\right)\right)}^{2} \theta^{-2}+\|u\|_{L_{\infty}\left(0, T ; \dot{B}_{\infty, \infty}^{-1}\left(\mathbb{R}^{3}\right)\right)}^{6} \theta^{-11}\right\} .
$$

Standard iteration gives us that for $0<r \leq \frac{1}{2}$,

$$
\mathcal{E}(r) \leq c\left(r^{\frac{1}{2}} \mathcal{E}(1)+\|u\|_{L_{\infty}\left(0, T ; \dot{B}_{\infty}^{-1, \infty}\left(\mathbb{R}^{3}\right)\right)}^{2}+\|u\|_{L_{\infty}\left(0, T ; \dot{B}_{\infty, \infty}^{-1}\left(\mathbb{R}^{3}\right)\right)}^{6}\right) .
$$

Taking into account (2.4), we get in addition that

$$
C\left(z_{0}, r\right) \leq c\left(r^{\frac{1}{2}} \mathcal{E}(1)+\|u\|_{L_{\infty}\left(0, T ; \dot{B}_{\infty, \infty}^{-1}\left(\mathbb{R}^{3}\right)\right)}^{6}\right) .
$$

This completes the proof of Theorem 1.2

\section{Appendix A. Proof of Lemma 2.2 .}

We let $w(\cdot, t)=S(t) f(\cdot)$ and $w_{\varphi}(\cdot, t)=S(t) \varphi f(\cdot)$, where $S(t)$ is a solution operator of the Cauchy problem for the heat equation with the initial data $f$ and $\varphi f$, respectevely. Then $u:=w \varphi-w_{\varphi}$ satisfies the equation

$$
\partial_{t} u-\Delta u=-2 \operatorname{div}(\nabla \varphi w)+w \Delta \varphi
$$

and the initial condition $u(\cdot, 0)=0$. A unique solution to the problem is as follows:

$$
u(x, t)=I+J,
$$

where

$$
\begin{gathered}
I=-\int_{0}^{t} \int_{\mathbb{R}^{3}} \Gamma(x-y, t-\tau)(2 \operatorname{div}(\nabla \varphi w))(y, \tau) d y d \tau, \\
J=\int_{0}^{t} \int_{\mathbb{R}^{3}} \Gamma(x-y, t-\tau)(w \Delta \varphi)(y, \tau) d y d \tau,
\end{gathered}
$$

and $\Gamma$ is the heat kernel.

Let us evaluate $I$. We abbreviate

$$
A:=\|f\|_{\dot{B}_{\infty, \infty}^{-1}\left(\mathbb{R}^{3}\right)}=\sup _{t>0} \sqrt{t}\|w(\cdot, t)\|_{L_{\infty}\left(\mathbb{R}^{3}\right)}
$$

and $\Omega=\operatorname{spt} \varphi$. Then we have

$$
\begin{aligned}
\sqrt{t}|I| & \leq 2 A \sqrt{t} \int_{0}^{t} \frac{1}{\sqrt{\tau}} \int_{\Omega} \frac{1}{(4 \pi(t-\tau))^{\frac{3}{2}}} \exp \left\{-\frac{|x-y|^{2}}{4(t-\tau)}\right\} \frac{|x-y|}{t-\tau} d y d \tau \leq \\
& \leq c A \int_{0}^{t} \sqrt{\frac{t}{\tau}} \frac{1}{(t-\tau)^{2}} \int_{\Omega} \exp \left\{-\frac{|x-y|^{2}}{4(t-\tau)}\right\} \frac{|x-y|}{\sqrt{t-\tau}} d y d \tau=
\end{aligned}
$$




$$
=c A\left[\int_{0}^{\frac{t}{2}} \ldots+\int_{\frac{t}{2}}^{t} \ldots\right]=c A\left(I_{1}+I_{2}\right) .
$$

Regarding $I_{1}$, consider first the case $0<t<1$. By the standard change of variables, we have

$$
I_{1} \leq c A C_{0} \int_{0}^{\frac{t}{2}} \sqrt{\frac{t}{\tau}} \frac{1}{\sqrt{t-\tau}} d \tau
$$

with

$$
C_{0}=\int_{\mathbb{R}^{3}} \exp \left\{-|u|^{2}\right\}|u| d u .
$$

And thus $I_{1} \leq c A$. In the second case $t \geq 1$,

$$
I_{1} \leq c|\Omega| \int_{0}^{\frac{t}{2}} \sqrt{\frac{t}{\tau}} \frac{1}{(t-\tau)^{2}} d \tau \leq c|\Omega| \frac{1}{t} \leq c|\Omega| .
$$

Now, let us evaluate $I_{2}$. Obviously,

$$
I_{2} \leq c \int_{\frac{t}{2}}^{t} \frac{1}{(t-\tau)^{2}} \int_{\Omega} \exp \left\{-\frac{|x-y|^{2}}{4(t-\tau)}\right\} \frac{|x-y|}{\sqrt{t-\tau}} d y d \tau .
$$

Make change of variables $\vartheta=t-\tau$, then

$$
\begin{gathered}
I_{2} \leq c \int_{0}^{\infty} \frac{1}{\vartheta^{2}} \int_{\Omega} \exp \left\{-\frac{|x-y|^{2}}{4 \vartheta}\right\} \frac{|x-y|}{\sqrt{\vartheta}} d y d \vartheta= \\
=c \int_{0}^{1} \ldots+c \int_{1}^{\infty} \ldots=J_{1}+J_{2} .
\end{gathered}
$$

For $J_{1}$, we have

$$
J_{1} \leq c C_{0} \int_{0}^{1} \frac{1}{\sqrt{\vartheta}} d \vartheta \leq c C_{0} .
$$

Finally, $J_{2}$ is bounded as follows:

$$
J_{2} \leq c \int_{1}^{\infty} \frac{1}{\vartheta^{2}} d \vartheta \leq c|\Omega|
$$

The quantity $J$ is estimated in the same way. Lemma 2.2 is proved.

Acknowledgement The first author is supported by the grant RFBR 17-0100099-a. The second author thanks Professors Cheng He and Zhifei Zhang for helpful discussions. 


\section{REFERENCES}

[1] A. Cheskidov, R. Shvydkoy, On the regularity of weak solutions of the 3D Navier-Stokes equations in $B_{\infty, \infty}^{-1}$, Arch. Ration. Mech. Anal. 195 (2010) 159-169.

[2] L. Burke, Q. Zhang, A priori bounds for the vorticity of axially symmetric solutions to the Navier-Stokes equations, Adv. Differential Equations 15 (5-6) (2010) 531-560.

[3] D. Chae, J. Lee, On the regularity of the axisymmetric solutions of the Navier-Stokes equations, Math.Z. 239(4) (2002) 645-671.

[4] C.-C. Chen, R. M. Strain, H.-T. Yau, T.-P. Tsai, Lower bound on the blow-up rate of the axisymmetric Navier-Stokes equations, Int. Math. Res. Not. 2008 (2008) 1-31.

[5] C.-C. Chen, R. M. Strain, H.-T. Yau, T.-P. Tsai, Lower bound on the blow-up rate of the axisymmetric Navier-Stokes equations II, Comm. Partial Differential Equations 34 (2009) 203-232.

[6] H. Hajaiej, L. Molinet, T. Ozawa, B. Wang, Necessary and sufficient conditions for the fractional Gagliardo-Nirenberg inequalities and applications to Navier-Stokes and generalized boson equations, IN Harmonic analysis and nonlinear partial differential equations, 159-175, RIMS Kôkyûroku Bessatsu, B26, Res. Inst. Math. Sci. (RIMS), Kyoto, 2011.

[7] T. Hmidi, D. Li, Small $\dot{B}_{\infty, \infty}^{-1}$ implies regularity. Dyn. Partial Differ. Equ. 14 (2017) no. 1, $1-4$.

[8] T. Y. Hou, L. Zhen, C. Li, Global regularity of the 3D axi-symmetric Navier-Stokes equations with anisotropic data, Comm. Partial Differential Equations 33 (7-9) (2008) 1622-1637.

[9] T. Y. Hou, C. Li, Dynamic stability of the three-dimensional axisymmetric Navier-Stokes equations with swirl, Comm. Pure Appl. Math. 61 (2008) 661-697.

[10] Q. Jiu, Z. Xin, Some Regularity Criteria on Suitable Weak Solutions of the 3-D Incompressible Axisymmetric Navier-Stokes Equations, New Studies in Advanced Mathematics, Vol. 2 (International Press, Somerville, MA, 2003), pp. 119-139.

[11] G. Koch, N. Nadirashvili, G. Seregin, V. Sverak, Liouville theorems for the Navier-Stokes equations and applications, Acta Math. 203 (2009) 83-105.

[12] O. A. Ladyzhenskaya, On the unique global solvability to the Cauchy problem for the NavierStokes equations in the presence of the axial symmetry, Zap. Nauchn. Semin. LOMI 7 (1968) 155-177.

[13] M. Ledoux, On improved Sobolev embedding theorems, Mathematical Research Letters, 10 (2003) 659-669.

[14] Z. Lei, Q. Zhang, A Liouville theorem for the axially-symmetric Navier-Stokes equations, J. Funct. Anal. 261 (8) (2011) 2323-2345.

[15] Z. Lei, Q. Zhang, Structure of solutions of 3D axisymmetric Navier-Stokes equations near maximal points, Pacific J. Math. 254 (2) (2011) 335-344.

[16] Z. Lei, E. Navas, Q. Zhang, A priori bound on the velocity in axially symmetric NavierStokes equations, Comm. Math. Phys. 341 (1) (2016) 289-307.

[17] Z. Lei, Q. Zhang, Criticality of the axially symmetric Navier-Stokes equations, Pacific J. Math. 289 (1) (2017) 169-187.

[18] X. Pan, Regularity of solutions to axisymmetric Navier-Stokes equations with a slightly supercritical condition, J. Differential Equations 260 (12) (2016) 8485-8529.

[19] G. Seregin, V. Sverak, On type I singularities of the local axi-symmetric solutions ofthe Navier-Stokes equations, Comm. Partial Differential Equations 34 (2009) 171-201.

[20] G. Seregin, W. Zajaczkowski, A sufficient condition of regularity for axially symmetric solutions to the Navier Stokes equations, SIAM J. Math. Anal. 39 (2) (2007) 669-685.

[21] G. Seregin, A note on bounded scale-invariant quantities for the Navier-Stokes equations, J. Math. Sci. (N.Y.) 185 (5) (2012) 742-745.

[22] M. R. Ukhovskii, V. I. Yudovich, Axially symmetric flows of ideal and viscous fluids filling the whole space, J. Appl. Math. Mech. 32 (1968) 52-61.

[23] W. Wang, Z. Zhang, Regularity of weak solutions for the Navier-Stokes equations in the class $L^{\infty}\left(\mathrm{BMO}^{-1}\right)$, Commun. Contemp. Math. 14 (3) (2012) 1250020, $24 \mathrm{pp}$.

[24] D. Wei, Regularity criterion to the axially symmetric Navier-Stokes equations, J. Math. Anal. Appl. 435 (1) (2016) 402-413. 
Mathematical Institute, University of Oxford, Andrew Wiles Building, Radcliffe Observatory Quarter, Woodstock Road, Oxford OX2 6GG, United Kingdom

E-mail address: seregin@maths.ox.ac.uk

School of Mathematics and Information Science, Henan Polytechnic University, Jiaozuo, Henan 454000, P. R. China

E-mail address: daoguozhou@hpu.edu.cn 